A N N A L E S Annales de Bretagne et des Pays de l'Ouest

\title{
Chronique des archives du Grand Ouest, 2013-2014
}

\section{(2) OpenEdition}

1 Journals

Édition électronique

URL : http://journals.openedition.org/abpo/3267

DOI : $10.4000 / a b p o .3267$

ISBN : 978-2-7535-5040-7

ISSN : 2108-6443

Éditeur

Presses universitaires de Rennes

Édition imprimée

Date de publication : 22 avril 2016

Pagination : 227-268

ISBN : 978-2-7535-5038-4

ISSN : 0399-0826

\section{Référence électronique}

«Chronique des archives du Grand Ouest, 2013-2014», Annales de Bretagne et des Pays de l'Ouest [En ligne], 123-1 | 2016, mis en ligne le 22 avril 2016, consulté le 15 septembre 2020. URL : http:// journals.openedition.org/abpo/3267 


\title{
Chronique des archives du Grand Ouest 2013-2014
}

\author{
BRETAGNE \\ Archives départementales du Finistère
}

Fiche signalétique
Archives départementales du Finistère
Site de Quimper
Tallée Henri-Bourde-de-La-Rogerie, cité administrative de Ty Nay - 29000 QUIMPER
Téléphone : 02989591 91 - Télécopie : 0298953169
Annexe de Brest
1 rue Jean-Fouchet -29200 BREST
Téléphone : 0298031614 - Télécopie : 0298476940
e-mail : archives.departementales@cg29.fr
Site internet : http://www.archives-finistere.fr
Heures d'ouverture : site de Quimper, du lundi au vendredi de $8 \mathrm{~h} 30$ à $17 \mathrm{~h} 00$
(dernière levée des documents à $16 \mathrm{~h} 00$ ). Fermeture le dernier vendredi du mois.
site de Brest, lundi-mercredi de $9 \mathrm{~h} 00$ à $12 \mathrm{~h} 00$ et de $13 \mathrm{~h} 00$ à $17 \mathrm{~h} 00$ (dernière levée
des documents à $16 \mathrm{~h} 30$ ). Fermeture les jeudis et vendredis

\section{1) Enrichissement des collections}

\section{Archives publiques}

Parmi les versements réalisés en 2013 et 2014, on peut noter les ensembles suivants :

- Registres des conservations des hypothèques de Morlaix (6 Q), Quimper premier bureau (7 Q), Quimper deuxième bureau (8 Q).

- Poursuite des versements concernant les affaires maritimes (quartiers de Camaret et Audierne, station de Roscoff). 
- Dossiers des ponts-et-chaussées concernant les ports du Finistère (1818-1945), la construction du pont Albert-Louppe et le pont suspendu de Paluden (1890-1940).

- Versements concernant le canal de Nantes à Brest (1806-1940).

- Reprise de la collecte des archives notariales en 2014, concernant des études pour lesquelles la collecte n'avait pas été réalisée depuis longtemps.

\section{Archives privées}

Plusieurs fonds d'archives privées entrés en 2013 et 2014 méritent d'être signalés.

$235 \mathrm{~J}$ - fonds Jean Bordereau, architecte (cet architecte a notamment travaillé sur l'aménagement du quartier du Braden à Quimper et à Port-laForêt).

$236 \mathrm{~J}$ - fonds de la coopérative maritime L'Abeille de Douarnenez, récupéré en vrac, in extremis, et concerne l'activité de cette coopérative maritime qui était située sur le port du Rosmeur à Douarnenez.

$237 \mathrm{~J}$ - fonds Joseph Halléguen, ancien maire de Quimper et député, partagé entre les Archives du Finistère et les Archives municipales de Quimper.

$240 \mathrm{~J}$ - fonds Georges Plouhinec, ancien militaire.

$242 \mathrm{~J}$ - fonds Yves Hénaff, architecte à Douarnenez, ayant beaucoup travaillé sur Douarnenez et Quimper.

On signalera également les documents entrés à l'occasion de la Grande Collecte des archives privées de la guerre 1914-1918, à laquelle les Archives du Finistère ont participé. Cette collecte a permis de faire rentrer des documents originaux, mais également de réaliser des copies numériques de documents, qui ont fait l'objet d'une mise en ligne. On pourra citer, parmi les originaux, les documents concernant Hervé Kergoat, originaire de Morlaix (1 J 1078, numérisés, consultables en ligne), le sous-lieutenant Tachet (1 J 1080), et parmi les documents numérisés, les documents concernant Jean Bonaventure, de Brest (1 Num 26).

\section{Archives iconographiques et audiovisuelles}

Les Archives du Finistère poursuivent leurs acquisitions de cartes postales, photographies, dessins. 1114 documents iconographies ont été acquis en 2013, et 1183 en 2014.

\section{2) Instruments de recherche, publications, bases de données}

Les Archives départementales du Finistère n'ont pas publié d'instruments de recherche au format papier, mais continuent de poursuivre leur effort de normalisation et de publication des instruments de recherche électroniques.

On citera notamment la rétroconversion de l'inventaire $97 \mathrm{~J}$ (fonds Charles Chassé), désormais consultable en ligne, et celle des bordereaux 
de versement des tribunaux d'instance et de grande instance, enrichissant le répertoire numérique des fonds des juridictions postérieurs à 1940 .

Les Archives du Finistère ont publié en novembre 2014 un ouvrage consacré à la Première Guerre mondiale, Aux Sources de la Grande Guerre, mettant en valeur pour une large part les documents issus de la Grande Collecte. Cet ouvrage présente une sélection de documents pour lesquels figurent la reproduction du document, une courte analyse et une transcription.

\section{3) Échos et nouvelles}

\section{Site internet}

Les années 2013 et 2014 ont vu le développement du site Internet avec la mise en ligne de nombreux documents numérisés, de nouveaux instruments de recherche et divers contenus éditoriaux (expositions virtuelles, zoom sur un document, aides à la recherche).

Pour 2013, la consultation du site représente 2652000 pages vues et 369480 visites, et en 2014, 2056000 pages vues pour 364500 visites. On notera donc une diminution de la fréquentation, malgré la mise en ligne de nouveaux documents numérisés (dénombrements de population, registres matricules militaires), mais toujours avec une offre réduite concernant l'état civil.

\section{Fréquentation de la salle de lecture}

La fréquentation de la salle de lecture a bien diminué en 2013 : le nombre total de communications est passé de 33246 en 2012 à 19566 en 2013. Cela s'explique par l'interruption de la communication d'un grand nombre de documents, en raison de l'état de contamination qui touche les archives. Les Archives du Finistère comptaient 1803 lecteurs inscrits en 2013 et 1709 en 2014. Toutefois, on note pour 2014 une légère remontée des communications, au nombre de 20582, les usagers s'orientant vers de nouveaux types de documents (hypothèques et enregistrement notamment).

\section{Archivage électronique}

En 2014 a été déployée la plate-forme d'archivage électronique As@lae, destinée à des tests en interne pour le Conseil départemental du Finistère. Parallèlement, le Conseil départemental dispose d'une plate-forme de préproduction dans le cadre du projet de service régional d'archivage électronique de e-mégalis en Bretagne depuis 2013.

Les Archives du Finistère poursuivent par ailleurs leurs actions de sensibilisation à l'archivage électronique auprès des services producteurs, et participent à divers projets de dématérialisation. 


\title{
Numérisation
}

En interne, l'atelier de numérisation du service numérise de nombreuses petites pièces isolées d'archives et de documents iconographiques. On pourra citer notamment les fonds Marcel Chevret, 36 Fi (photographies aériennes de quelques villes et côtes du Finistère, 1985-1986) et Perardel, 35 Fi (Photographies de paysages du nord du Finistère et de la presqu'île de Crozon, 1903-1909).

Les registres matricules militaires des classes 1867-1891 ont commencé à être numérisés. Les classes 1867 à 1891 ont été réalisées en 2013 et les classes 1892 à 1903 en 2014, ainsi qu'un complément constitué des classes 1860 à 1866. 1931.

Les dénombrements de population ont été numérisés jusqu'à l'année

\section{Service éducatif}

Les Archives départementales du Finistère disposent depuis la fin de l'année 2013 d'un professeur conseiller relais, qui a notamment participé à l'élaboration de l'outil pédagogique sur la Résistance dans le Finistère, publié en 2014.

Malgré la limitation des visites dues aux problèmes de contamination, le service a accueilli, en 2014, 84 élèves de lycée et 30 étudiants.

\section{Archives départementales d'Ille-et-Vilaine}

\author{
Fiche signalétique
}

Archives départementales d'Ille-et-Vilaine

Adresse postale : Direction des Archives et du Patrimoine

1, rue de la préfecture, CS 2418 - 35041 ReNNES Cedex 1

Adresse topographique : 1 rue Jacques-Léonard - 35000 RENNES

Téléphone : 0299024000 - Télécopie : 0299024001

E-mail : archives@cg35.fr

Site internet : $w w w$.archives 35 .fr

Heures d'ouverture : du lundi au vendredi de $9 \mathrm{~h} 00$ à $17 \mathrm{~h} 00$

(sauf le $1^{\text {er }}$ lundi de chaque mois)

(Fermeture annuelle la première quinzaine d'août)

Pour une première approche des fonds, consulter :

Guide des archives d'Ille-et-Vilaine, tome 1, volume 1,1994

(fonds antérieur à la Révolution) et le site internet (rubrique Rechercher) 


\section{1) Enrichissement des collections}

\section{Archives publiques}

En 2013 et 2014, le ralentissement des entrées d'archives publiques contemporaines déjà constaté les 2 années précédentes se poursuit : 612 m linéaires en 2013 et 614 m linéaires en 2014 pour les entrées en W, W-dépôt, H-dépôt et ETP.

L'accent est toujours mis sur les missions de conseil, de formation, d'expertise et de contrôle auprès des producteurs d'archives publiques, pour limiter la collecte en raison de la saturation prochaine des magasins équipés. Ainsi l'année 2013 a vu l'installation d'un comité de pilotage pour la mutualisation de la fonction archives dans les services de l'État avec pour objectif de définir les priorités au sein des 4 départements bretons. Les Archives d'Ille-et-Vilaine ont également été actives au sein de groupes de travail tels que le groupe régional piloté par le Conseil national pour l'accès aux origines personnelles et l'Agence régionale de la santé sur la problématique de la préservation des données relatives aux naissances dans le secret; le groupe régional sur la protection maternelle et infantile; le groupe régional sur les archives de Pôle Emploi. En 2014, les travaux d'élaboration de tableaux de gestion et chartes d'archivage ont été poursuivis telle celle de la DRAAF.

En matière de dématérialisation, on peut noter l'élaboration et la signature d'un document cadre pour la numérisation des dossiers de titres de séjour à la préfecture d'Ille-et-Vilaine, permettant d'autoriser la destruction des dossiers papier.

En ce qui concerne les établissements publics, on peut signaler la découverte, lors d'une visite au Centre hospitalier de Vitré en 2013, d'archives remontant au début du XIX ${ }^{\mathrm{e}}$ siècle comprenant notamment des registres d'entrée, un fichier des soldats blessés en 1914-1918, des cahiers de maternité. Pour 2014, on peut remarquer l'entrée des fonds des chambres de commerce (200 m linéaires), notamment le fonds historique de la chambre de commerce de Saint-Malo.

Le groupe de travail réunissant, depuis 2010, les archivistes chargés des archives contemporaines dans les quatre services d'Archives départementales a poursuivi ses travaux, en vue de l'harmonisation des pratiques des différents services notamment tant au niveau de l'archivage papier que de l'archivage électronique.

Les visas d'élimination réglementaire ont été donnés en 2013 pour 10545 m linéaires d'archives publiques (hors communes) et en 2014 pour 5834 m linéaires, auxquels il faut ajouter les archives du Pôle interrégional d'apurement administratif situé à Rennes, soit 25478 m linéaires (en 2013) et 5559 m linéaires (en 2014).

En matière d'archives antérieures à 1940, on notera la restitution par la ville de Vitré d'un dépôt de 42 m linéaires. de publications de mariage de l'arrondissement de Vitré (intégration en sous-série $3 \mathrm{E}$ ). La politique de collecte des archives notariales s'est poursuivie et a permis l'entrée de 23 nouveaux versements. 


\section{Archives privées}

Les principales entrées en 2013 et 2014 sont le fonds de l'Association régionale pour l'animation du Parlement de Bretagne (ARAP), les fonds d'architectes Marc Petitjean, Hervé Chouinard, Joël Gautier et Patrice Simon, le fonds du militant agricole Paul Havard, celui de Max Jonin, secrétaire général de Bretagne vivante-Société pour l'étude et la protection de la nature en Bretagne (SEPNB) de 1973 à 2000, puis président de la Société géologique et minéralogique de Bretagne, des archives des partis politiques CDS, UDF et MODEM d'Ille-et-Vilaine, le fonds de l'ancien directeur des Archives départementales Jacques Charpy, le fonds de l'Association des musées de société de Bretagne Buhez, le fonds d'archives familiales et seigneuriales Rolland du Noday, le fonds du Théâtre de poche de Hédé et de l'Association «Bruit de lire " (Goncourt des lycéens), sans omettre plusieurs compléments de fonds (Jean-François Botrel, CREAI (Centre régional pour l'enfance et l'adolescence inadaptées), François Corre, Michel Denis, IRPA (Institut régional du patrimoine/Bretagne), Hay des Nétumières), ni les documents, des lettres notamment, de combattants des deux guerres mondiales, dans le sillage de l'opération " grande collecte ". Il faut aussi signaler l'entrée de nombreux travaux universitaires sur la Bretagne contemporaine issus du fonds Michel Denis (sous-série 2J).

\section{Fonds iconographiques}

Parmi les 3000 documents entrés en 2013 et 2014 aux Archives départementales, citons un joli fonds de cartes postales, tirages et négatifs souples sur la Bretagne des années 1936 à 1960) (fonds Goguet, 60 Fi), un fonds de photographies aériennes (négatifs de moyen format) des années 1980 sur les principales villes du département (fonds Chevret, $59 \mathrm{Fi}$ ), un fonds de négatifs de moyen format du photojournaliste Jean Ribière (53 Fi) ou encore un petit fonds d'affiches et de tracts sur le référendum du 29 mai 2005 sur l'Europe (fonds Géraud, 58 Fi).

Dans le cadre de la préparation des commémorations de la Grande Guerre, acquisition d'un fonds de photographies contemporaines du photographe Jean Richardot sur les paysages fréquentés par les poilus d'Illeet-Vilaine sur le front pendant le conflit (fonds Richardot, 57 Fi).

\section{Bibliothèque}

La bibliothèque a continué de s'enrichir (270 ouvrages achetés en 2013 et 322 en 2014) et a reçu 101 revues scientifiques.

\section{2) Instruments de recherche}

En 2013, plus de 600 m linéaires de fonds postérieurs à 1940 ont été classés. On note toujours l'importance des fonds judiciaires (plus de 
$121 \mathrm{~m}$ linéaires); le tout aussi important chantier entrepris sur les fonds de la DRIRE (plus de 90 m linéaires) : sont notamment concernées les mines et carrières ainsi que l'énergie (dont les barrages); ou encore, le traitement des plans minutes de remembrement (1941-1983).

En 2014, plus de 700 m linéaires ont été traités dont une part toujours importante de fonds judiciaires (400 m linéaires). On peut signaler les fonds de l'École nationale supérieure d'architecture (23 m linéaires).

Pour les archives antérieures à 1940, le traitement de la série R (affaire militaires, 1800-1940) a été achevé. Ce sont ainsi plus de $300 \mathrm{~m}$ linéaires de documents qui ont été classés et ont fait l'objet d'un instrument de recherche analytique.

Traitement d'un intéressant versement effectué en 2012 par l'Établissement national des invalides de la marine (ENIM). Les 20 m linéaires de ce fonds complètent le fonds de l'inscription maritime déjà conservé (quartiers de Saint-Malo et de Dinan) et contient également des dossiers de pension des marins.

Concernant les archives notariales, les années 2013 et 2014 ont permis de livrer 23 nouveaux instruments de recherche, concernant $425 \mathrm{~m}$ linéaires. de minutes et répertoires des actes de plus de 75 ans.

Pour l'iconographie, outre l'alimentation régulière de la base de données par les entrées de documents à l'unité (cartes postales, cartes et plans, gravures, affiches), plusieurs instruments de recherche ont été réalisés; citons notamment le fonds du graphiste Desailly (mise à jour annuelle en fonction des nouvelles affiches données), le fonds photographique Bartoli (51 Fi), le fonds du photographe Simon (47 Fi) ou encore le fonds de Monti (51 Fi) relatif à l'activité grainetière dans le pays de Louvigné-du-Désert.

En matière d'archives privées, la plupart des fonds entrés, sauf les fonds d'architectes, ont fait l'objet d'inventaires peu de temps après leur entrée. L'important fonds d'archives de la coopérative ouvrière de graniterie L'avenir de Louvigné-du-Désert (165 J, 1906 à 1985, près de 100 m linéaires) a fait l'objet d'un classement très détaillé.

Pour les archives communales, celles des communes de Saint-Étienneen-Coglès et Saulnières en 2013 (env. 100 m linéaires) et, en 2014, d'Availlessur-Seiche, Chauvigné, Etrelles, Fleurigné, Lillemer, Le Petit-Fougeray, Pipriac, Saint-Malo-de-Phily, Saint-Marc-sur-Couesnon, Saint-Pern, Tresbœuf, La Selle-Guerchaise, et de 4 structures intercommunales : communauté de communes du canton de Pipriac et de Brocéliande, SMICTOM des Forêts et syndicat intercommunal d'assainissement du Val de Seiche et de l'Ise (env. 420 m linéaires) ont été dotées d'un instrument de recherche règlementaire.

\section{3) Échos et nouvelles}

\section{Service éducatif}

Le service éducatif des Archives départementales a reçu 1126 élèves en 2013 et 1147 en 2014, de l'école primaire au lycée pour des visites du 
bâtiment et des ateliers pédagogiques, animés par trois professeurs (arts plastiques, histoire, français). Il a continué de proposer diverses ressources aux enseignants, l'atelier calligraphie confirmant son attrait.

\section{Fréquentation de la salle de lecture}

En 2013 et 2014, la salle de lecture a respectivement accueilli 1868 et 1844 lecteurs, ce qui a occasionné 6264 et 6302 séances de travail ainsi que 24896 et 21795 communications d'archives. Périodiques, ouvrages, microfilms, documents numériques, sonores ou audiovisuels ne sont pas comptabilisés. Parallèlement à cette activité, 1254 et 1277 recherches par correspondance ont été traitées avec une forte proportion de demandes liées au droit du sol (entre 30 et $40 \%$ du total).

\section{Internet et nouvelles technologies}

La présence des Archives départementales d'Ille-et-Vilaine sur le web se confirme depuis l'ouverture en 2012 d'un compte twitter @archives35 avec aujourd'hui (octobre 2015) 607 abonnés. La page Facebook www.facebook. com/archives35, lancée depuis l'automne 2011, compte aujourd'hui 1966 amis qui suivent assidument l'actualité du service par ce biais. L'activité du site internet $w w w$.archives35.fr a été équivalente aux années passées avec près de 178000 et 112000 visiteurs uniques sur nos pages en 2013 et 2014 (l'installation d'un nouvel outil de gestion des contenus a provoqué l'interruption des statistiques de fréquentation du site entre le ler avril et le 1er juin 2014). Le Département a poursuivi un partenariat annuel avec la radio RCF Alpha pour une émission hebdomadaire de 13 minutes intitulée " Mag'histoire ». Un documentaire sur l'architecture du bâtiment des AD35 "A la mesure du temps " a été réalisé par la société lyonnaise " On stage ».

\section{Numérisation}

Numérisation des registres matricules militaires (1859-1940), des tables de successions et absences de l'enregistrement (1935-1969) et de près de 14000 images (dont des documents iconographiques privés en lien avec la Première Guerre mondiale ou des diapositives de la CAOA d'Ille-et-Vilaine en rapport avec les objets mobiliers protégés au titre des MH), du Semeur d'Ille-et-Vilaine, organe officiel de l'Union départementale des syndicats CGT d'Ille-et-Vilaine, publié de 1919 à 2001, des états de sections du cadastre des communes d'Ille-et-Vilaine, avec mise en ligne des registres matricules et de complément d'état civil (Saint-Malo).

Mise en ligne des images numérisées des recensements de population (sous-série $6 \mathrm{M}$ ), des registres matricules (sous-série $1 \mathrm{R}$ ), de plus de 12000 cartes postales anciennes relatives à l'Ille-et-Vilaine (sous-série 6 Fi) et de nombreux instruments de recherche. 


\section{Exposition}

En 2013, a eu lieu le 6e rendez-vous du cycle "Bande dessinée et histoire " : Août 44 : Saint-Malo dans la bataille, autour de la bande dessinée Cézembre de Nicolas Malfin. Le cycle Granit en Ille-et-Vilaine, lancé en 2012, s'est prolongé par deux expositions, l'une à Monthault, avec la publication du 4 e livret de la collection "Archives \& histoire ", Au pays du granit avec Paul Lebois et son roman La villa des bagnards, et l'autre à Saint-Pierre-dePlesguen, avec la publication du volume 2 de la collection « Patrimoine écrit d'Ille-et-Vilaine ", l'histoire inédite de la commune rédigée par l'instituteur François Garnier.

La programmation 2014, centrée autour de la commémoration du centenaire de la première guerre mondiale, a donné lieu à plusieurs manifestations et publications :

- l'exposition "Le Front, l'arrière, la mémoire ", accompagnée d'un ouvrage intitulé Hommes et femmes d'Ille-et-Vilaine dans la Grande Guerre, sous la direction d'Eric Joret et Yann Lagadec, en coédition avec la Société archéologique et historique d'Ille-et-Vilaine

- le $7^{\mathrm{e}}$ rendez-vous du cycle $\mathrm{BD}$, " 14-18 la vie à l'Arrière "

- «14-18. De la guerre aux paysages d'aujourd'hui ». Exposition de photographies contemporaines de Jean Richardot, dont une version itinérante a été tirée.

- La Grande Guerre : Archives \& histoire n5, livret qui a accompagné une exposition itinérante réalisée dans les communes de Roz-sur-Couesnon et Vieux-Vy-sur-Couesnon.

\section{Archives départementales du Morbihan}

\section{Fiche signalétique}

Archives départementales du Morbihan

80 rue des Vénètes -56010 VANNES cedex

Téléphone : 0297463252

e-mail : archives@morbihan.fr

Site internet : $w w w$.archives.morbihan.fr

Heures d'ouverture : du lundi au vendredi $9 \mathrm{~h} 00-17 \mathrm{~h} 30$

Fermeture mensuelle le $2^{\mathrm{e}}$ mercredi matin de chaque mois

Fermeture annuelle du 15 au 31 décembre 


\section{1) Enrichissement des collections}

\section{Archives publiques}

En 2013-2014, un total de 2228 m linéaires d'archives publiques sont entrées aux Archives départementales du Morbihan (2013: $1501 \mathrm{~m}$ linéaires, 2014 : 727 m linéaires). Il est à noter que 31 fonds d'archives communales (2013 : 7, $2014: 24)$ ont été déposés représentant 166 m linéaires (2013 : 41 m linéaires, 2014 : 125 m linéaires).

Parmi les versements et dépôts pris en charge sur cette période, on peut noter les ensembles suivants :

- 3 ES 33 : fonds de la commune de Carentoir : registres paroissiaux et documents d'administration communale (1541-1970).

- $4 \mathrm{E}$ : registres d'état civil versés par les tribunaux de grande instance de Vannes et Lorient (1907-1937).

- 1907 et $1913 \mathrm{~W}$ : archives relatives au remembrement versées par la Direction départementale des territoires et de la mer : procès-verbaux et dossiers administratifs et techniques (1950-2011).

- $1922 \mathrm{~W}$ : archives du quartier maritime d'Auray (1939-2003).

- 9 H-dépôt : fonds du centre hospitalier Bodélio de Lorient (1811-2004);

- Série U, 2018-2026 W : archives du tribunal de grande instance de Vannes : minutes et dossiers de procédure (1894-2008).

- $1423 \mathrm{~W}, 1999-2003 \mathrm{~W}$ : registre du commerce du tribunal de commerce de Lorient (1943-2001).

- 1998 W : archives du commissariat de police d'Hennebont (1945-1997).

\section{Archives privées}

En 2013-2014, 136 m linéaires d'archives privées ont intégré les collections des Archives départementales (2013: 7 m linéaires, 2014 : 129 m linéaires).

Parmi les fonds collectés, il faut signaler :

- Fonds Yann-Fañch Kemener (198 J) : chanteur, écrivain et musicologue, son fonds reflète son activité d'artiste et de chercheur dans le domaine de la culture et de la musique traditionnelle bretonne (1970-2014).

- Mémoires d'Auguste Leson (199 J) : Journal tenu entre 1919 et 1944 par Auguste Leson, réalisateur et exploitant du barrage de Guerlédan.

- Fonds de l'association Breizh Santel (211 J) : fonds d'une association dédiée à la sauvegarde du patrimoine religieux (1952-2010).

- Fonds Géniaux (71 Fi) : 30 photographies prises par Paul Géniaux dans le département en 1895.

- Fonds Pugeault (74 Fi) : 2000 plaques de verre prises par le Préfet Emile Pugeault et sa famille dans le département et sur ses lieux d'exercice (Indre, Lozère, Doubs) entre 1880 et 1918. 


\section{2) Instruments de recherche et publications}

En 2013-2014, 2165 m linéaires ont été classés (2013 : 1123 m linéaires, 2014 : 1042 m linéaires) et 207 instruments de recherches ont été produits (2013: 77, $2014: 130)$.

Sur ce total, les archives contemporaines représentent $1647 \mathrm{~m}$ linéaires et 168 instruments de recherche qui ont été mis en ligne sur le moteur de recherche des Archives départementales, parmi ceux-ci on peut citer :

- les fonds des quartiers maritimes du département (101 W, $1922 \mathrm{~W}$, 1923 W, 1741 W, 1742 W); le fonds de l'hôpital Bodélio de Lorient (9 H-dépôt 2 et 3 ).

- le fonds de la direction départementale du ravitaillement général $(1430 \mathrm{~W})$.

- le fonds de la mission photographique du ministère de la reconstruction et de l'urbanisme (104 W, $1804 \mathrm{~W})$.

Pour les autres séries, un instrument de recherche sur les registres d'état-civil collectés pour la période 1906-1936 a été publié et la série R (affaires militaires, 1800-1940) a fait l'objet en 2013 d'un récolement complet. Un travail de classement a été entamé ou poursuivi sur les fonds suivants :

- sénéchaussée de Rhuys (7 B, XVI ${ }^{\mathrm{e}}$-XVIII ${ }^{\mathrm{e}}$ siècles);

- préparation militaire et recrutement de l'armée (1 R, 1800-1940);

- anciens combattants et victimes de guerre (3 R, 1800-1940);

- mer, transports, transports maritimes (4 S, 1800-1940);

- service hydraulique, associations syndicales autorisées (7 S, 18001940);

- établissement public de santé mentale de Saint-Avé (20 H-dépôt).

Les instruments de recherche correspondant devraient être publiés en 2015-2016.

\section{3) Échos et nouvelles}

\section{Expositions}

Trois expositions ont été présentées en 2013. L'exposition Sur les rails du Morbihan, inaugurée en 2012 s'est terminée le 19 avril 2013 et a accueilli en 20131102 visiteurs.

Une brève exposition des clichés du fonds Paul Lotz a été présentée du 24 mai au 10 juin 2013 (78 visiteurs), dans le sillage de la présentation de l'ouvrage publié sur ce fonds. Cet ouvrage intitulé Photographies Lotz, l'album d'un gentleman farmer présente une sélection de photographies prises entre 1890 et 1930 par Paul Lotz et les membres de sa famille. Ces clichés reviennent sur la vie familiale des Lotz et les voyages qu'ils ont effectués en France. Elles présentent également l'activité d'ingénieur agronome de Paul Lotz au début du xxe siècle.

L'exposition photographique Belle-Île-en-Mer 1930-1960 : rêves et réalités a attiré 573 visiteurs du 17 juin au 18 octobre. 
En 2014, une exposition multimédia Les Morbihannais dans la guerre 14-18, a été ouverte au public à partir du 20 septembre. Cette exposition avait pour objectif de revenir sur la manière dont les habitants du département ont vécu le conflit sur le front aussi bien qu'à l'arrière.

Un catalogue de 252 pages a été publié pour prolonger l'expérience offerte par l'exposition.

Cette exposition a réuni en 20142372 visiteurs dont 1149 scolaires pour trois mois d'exposition.

Parallèlement, les Archives mettent gratuitement à disposition des établissements scolaires et des organismes culturels et éducatifs les expositions préalablement présentées dans leur hall. En 2013-2014, les Archives du Morbihan ont ainsi réalisé 80 prêts d'expositions (2013 : 33, 2014 : 47). Près de 38393 personnes les ont visitées sur la période $(2013: 24313,2014$ : 14080).

Le premier numéro de la lettre d'information des Archives départementales, intitulée Les coulisses de l'histoire, est paru en juillet 2013. Cette revue paraît deux fois par an en juillet et en janvier.

\section{Activités pédagogiques}

Le service éducatif sensibilise un grand nombre d'élèves morbihannais au travers de multiples activités sur site (ateliers, travaux sur documents, visites du bâtiment et des expositions...) ou hors les murs (archivobus dont les activités ont repris en 2013 avec pour objectif d'organiser des ateliers et séances de travaux dirigés dans les collèges, expositions itinérantes). Ainsi, pour la période, un total de 8898 élèves (2013 : 3315, 2014 : 5583) a pu bénéficier de l'action pédagogique des Archives départementales.

En 2014, un parcours intitulé Enquêtes d'archives a été bâti afin de faire découvrir les sources juridiques et fiscales conservées aux Archives départementales à des étudiants en L2-L3 de droit à l'Université de Bretagne Sud (Vannes). Cet enseignement a été intégré dans une unité optionnelle du cursus.

\section{Fréquentation de la salle de lecture - site Internet}

La fréquentation de la salle de lecture a connu en 2013 un léger rebond après plusieurs années de baisse. Le nombre de lecteurs inscrits ou réinscrits est demeuré en légère baisse mais le nombre de séances et de communications de documents en salle sont reparties à la hausse avec respectivement 20826 et 4445 unités. L'année 2014 a été l'occasion de réaménager une partie des espaces dédiés au public au deuxième étage du bâtiment. L'ancien espace multimédia a réintégré la salle de lecture dont la présidence de salle et la banque d'accueil ont été repensés afin d'offrir une meilleure accessibilité. Les usuels qui étaient installés sur des rayonnages en mezzanine ont été réimplantés de plain-pied pour la même raison. En raison de ces travaux la salle de lecture a été partiellement fermée de fin avril à fin 
octobre 2014, le léger rebond de fréquentation observé en 2013 n'a donc pas pu être confirmé.

Des ateliers lecteurs visant à présenter les fonds récemment classés ou utiles pour certains types de recherche (recherches foncières, généalogie...) ont été initiés en 2014.

La fréquentation du site Internet des Archives départementales a en revanche connu une progression spectaculaire : un peu plus de 250000 visiteurs en 2013 pour 969000 connexions et 383000 visiteurs en 2014 pour environ 2700000 connexions. L'offre internet des Archives départementales a évolué sur cette même période : une nouvelle version du moteur de recherche a été proposée à partir de novembre 2013 ainsi qu'un nouvel outil de visualisation des documents numérisés. En août 2014, le site internet dans son ensemble a été refondu afin de proposer de nouvelles rubriques (zooms sur des épisodes de l'histoire du département, contenus à destination des producteurs d'archives...) et une navigation plus conviviale et intuitive.

Une première tranche de registres matricules numérisés (classes 18671912) a été mise en ligne au début de l'année 2014.

\section{Numérisation}

Les Archives du Morbihan ont poursuivi en 2013-2014 leurs travaux de numérisation : la numérisation et l'indexation des registres matricules de recrutement militaire pour les classes 1913-1921 a été lancée en 2014 et doit s'achever en 2015.

Les archives des amirautés de Vannes et Lorient, les archives du consulat de Lorient, des subdélégations du département et une partie des archives de la commission intermédiaire des états de Bretagne, préalablement microfilmés, ont été numérisées.

Parallèlement, les travaux de numérisation en interne ont été développés et plusieurs ensembles ont été traités : fonds iconographiques (cartes postales, photos anciennes), reprise de la numérisation des listes de recensement de population (1805-1911). 


\section{Archives municipales de Rennes}

\begin{tabular}{|c|}
\hline Fiche signalétique \\
Archives municipales de Rennes \\
18, avenue Jules-Ferry, CS 63126 - 35031 RENNES cedex \\
Téléphone : 0223621260 - Télécopie : 0223621269 \\
e-mail : archives @ ville-rennes.fr \\
Site internet $:$ www. archives.rennes.fr \\
Heures d'ouverture : le lundi $13 \mathrm{~h} 30-17 \mathrm{~h} 00$ \\
mardi, mercredi et vendredi $9 \mathrm{~h} 00-12 \mathrm{~h} 30$ et $13 \mathrm{~h} 30-17 \mathrm{~h} 00$ \\
jeudi $9 \mathrm{~h} 00-17 \mathrm{~h} 00$ en continu
\end{tabular}

\section{1) Enrichissement des collections}

\section{Archives publiques}

L'effort de collecte des fonds en provenance des services municipaux et communautaires n'a pas faibli ces dernières années. Atteignant près de 500 m linéaires en 2014, les nouveaux fonds collectés reflètent l'actualité de la collectivité. Sans surprise, l'année 2014 aura permis de recueillir un important fonds politique consécutivement aux élections municipales. Le cabinet du maire sortant qui ne se représentait pas, Daniel Delaveau, mais aussi ceux de plusieurs adjoints qui faisaient de même après plusieurs mandats ont en effet procédé à d'importants versements préparés conjointement avec les archivistes.

Sur le plan culturel et patrimonial, on notera le versement émanant du musée de Bretagne qui complétera avantageusement le fonds d'archives du musée des Beaux-arts auquel il a été associé de ses débuts jusqu'à son déménagement sur le site actuel des Champs Libres.

La refonte de la carte intercommunale en Ille-et-Vilaine a conduit à l'extension du périmètre de la communauté d'agglomération de Rennes Métropole pour laquelle les Archives de Rennes sont compétentes par convention de mise à disposition de service. De ce fait, fin 2013, le fonds clos de la communauté de communes du pays de Bécherel dissoute a rejoint les rayonnages du service.

\section{Fonds privés}

Avec près de 100 m linéaires en 2014, les entrées par voie extraordinaire ont suivi la même tendance haussière que les fonds publics tout en cultivant une certaine diversité. On pourra ainsi trouver aux côtés des archives d'une pharmacie, celles d'une librairie spécialisée dans les arts qui a récemment cessé son activité ou encore, sous forme de don numérique, des papiers relatifs au fonctionnement d'une minoterie vieille de plusieurs siècles mais toujours active près du centre-ville. 
La prépondérance des fonds d'architectes parmi les archives privées sera augmentée par les dons de Patrice Simon et d'Étienne Chouinard, fils de Louis, qui avait lui-même fait don de ses archives professionnelles et auquel les Archives avaient rendu hommage à travers une exposition en 2004.

Le photographe professionnel Michel Ogier a complété son don couvrant la période des années 80 à 2000 qui vient considérablement enrichir le fonds iconographique des Archives de Rennes.

Enfin, les commémorations du centenaire de la Grande Guerre ont permis la collecte de petits fonds personnels avec notamment des lettres de poilus et autres livrets militaires.

\section{2) Échos et nouvelles}

\section{Bâtiment}

Le projet initial de relocalisation vers un nouveau site à construire a été suspendu. De nouvelles études seront lancées vraisemblablement dès 2018 pour permettre d'avancer sur cet épineux dossier, particulièrement dans le contexte de collecte abondante mentionné plus haut.

L'un des enjeux de l'année 2015 et des suivantes sera de maintenir un accès de qualité pour le public alors que l'avenue Jules-Ferry est désormais en impasse avec la mise en place du chantier de la ligne b du métro, une nouvelle station Jules-Ferry devant entrer en service début 2019 à $50 \mathrm{~m}$ de l'emplacement actuel des Archives.

\section{Salle de lecture et publics}

La fréquentation de la salle de lecture a crû de manière notable en 2013 (+ 101 lecteurs inscrits) pour un nombre de communications sensiblement supérieur à l'année 2012 (+ 111 articles). Ces variations sont particulièrement satisfaisantes, d'autant plus que les fonds les plus consultés (état civil, délibérations) sont disponibles en ligne depuis maintenant une décennie.

Ce regain d'activité n'est pas étranger au centenaire, en 2013, de la naissance de Georges Maillols, architecte rennais prolifique, dont les Archives conservent le fonds professionnel (14 Z). Ce fonds a été fortement sollicité tant par les auteurs d'un beau livre auquel les Archives ont contribué (Georges Maillols, architecte, Presses universitaires de Rennes, 2013) que par les étudiants de l'école nationale supérieure d'architecture de Bretagne, invités par leurs enseignants à redécouvrir cette architecture à l'occasion du centenaire du bâtisseur.

La collection des petits guides d'aide à la recherche historique s'est enrichie de deux nouvelles publications associées au programme d'expositions des Archives pour les années 2013 et 2014. C'est ainsi qu'après Sur les 
traces des commerces rennais consacré aux sources commerçantes, a été proposé Les archives s'en vont en guerre autour des documents militaires.

Les résultats de l'enquête nationale des publics d'archives dont les Archives de Rennes faisaient partie du panel ont confirmé les observations faites en interne. Sur la typologie du public notamment, la forte proportion de public scientifique, principalement étudiant (plus de $50 \%$ des inscrits en salle de lecture en 2014) est confirmée. L'étude révèle que la part majoritaire du public ayant répondu à l'enquête aux Archives de Rennes se situe dans la tranche des 18-25 ans (à 33 \%), à l'opposé de la tendance nationale où ce sont les séniors (66-75 ans) qui se dégagent à hauteur $26 \%$.

Le statut de cité universitaire de Rennes, couplé à la présence cumulée d'une école d'architecture et de nombreux fonds d'architectes aux Archives municipales, est un facteur d'explication.

\section{Nouvelles technonologies}

Devant l'ampleur des fonds touchant à la thématique du commerce et les multiples usages que l'on pouvait en espérer, il a été décidé le développement d'une application cartographique géo-localisée «Quand les commerces se montrent " permettant la mise en valeur de 4000 documents qui sont autant de notices déjà présentes dans la base de données. Celle-ci a vocation à s'enrichir au fur et à mesure des découvertes et du traitement de fonds sériels en relation avec le commerce.

\section{Expositions}

Dédiée à l'histoire du commerce dans la ville ("Quand le commerce se raconte "), l'exposition pour l'année 2013 s'est à nouveau déroulée à l'opéra. Ce projet d'envergure s'est notamment accompagné d'une collecte de témoignages d'acteurs du commerce rennais avec édition d'un livret. La réalisation d'un mini-site dédié a permis de prolonger l'exposition physique.

Sans grande originalité, l'exposition 2014 a été consacrée au centenaire de la Grande Guerre. Labélisée par la mission du Centenaire et intitulée « La guerre au loin ", elle a pris place, comme à l'habitude, dans le carré Lully de l'opéra de Rennes, place de Mairie où l'inconvénient principal réside en l'absence de comptage des visiteurs qui ne peut qu'être estimatif. Fait nouveau, un livret d'accompagnement a été édité. Il est d'ores et déjà disponible en lien avec l'exposition itinérante déclinée pour commémorer ce centenaire durant les prochaines années.

La création artistique "Mes chers parents " par la Compagnie théâtrale Les Becs Verseurs a permis la mise en valeur originale de la correspondance de guerre de Charles Oberthür conservée par les Archives. Présenté à deux reprises (16 et 17 mai 2014) au grand public et à six classes lors d'une représentation scolaire en matinée, dans la salle historique de la Cité en centre-ville de Rennes mais également à travers le département, ce 
spectacle a bénéficié du soutien du fonds d'accompagnement artistique et territorial du conseil général d'Ille-et-Vilaine.

\section{Action culturelle et médiation}

Le bon relais dans la presse locale mais également la presse gratuite (édition rennaise de 20 Minutes) des "Jeudis des Archives " assure une fréquentation soutenue des différents rendez-vous. La contribution régulière (1 numéro sur 2) des Archives aux pages " Mémoire " du magazine municipal bimestriel Les Rennais permet également une visibilité renforcée.

Cette programmation semble avoir trouvé son public puisque le nombre de personnes touchées a triplé depuis 2009, dernière année complète avec la précédente forme d'action culturelle.

La fréquentation du service éducatif a quant à elle plus que doublé sous le double effet d'une offre enrichie et d'une actualité particulièrement favorable autour de la Grande Guerre.

En 2014, c'est au total plus de 6600 personnes qui ont bénéficié de l'action culturelle des Archives sous toutes ses formes (expositions, service éducatif et "Jeudis des Archives" notamment).

\section{Partenariats}

Un nouveau partenariat a été conclu avec la radio rennaise Canal B pour l'animation d'une chronique mensuelle. À partir d'un document insolite, des archivistes sont invités à échanger avec le journaliste Ronan Le Mouhaër. Toutes les chroniques sont téléchargeables sur le site des Archives.

Sollicitées dans le cadre d'un partenariat entre la Ville de Rennes et la Fondation de France, les Archives ont reçu les membres de la chambre départementale des notaires d'Ille-et-Vilaine pour une visite guidée des locaux et de l'exposition consacrée au commerce. Le succès rencontré a permis d'envisager de nouvelles rencontres dès 2014 avec les membres de la fédération COBATY rassemblant les professionnels concernés par l'acte de bâtir, l'urbanisme et l'environnement. L'occasion de présenter les missions des Archives et la découverte des fonds touchant à leurs métiers.

Le partenariat avec la délégation Atlantique de l'Ina se poursuit et assure un succès renouvelé aux séances "Archives à l'écran ". Celle du 31 janvier 2013, consacrée au quartier rennais du Blosne, délocalisée dans ce quartier et mise en musique par les élèves des classes-orchestres, a drainé 450 spectateurs. Le partenariat sera étendu dès 2015 avec la mise à disposition d'une borne au sein de l'exposition "Sur un air de fêtes » où des extraits audiovisuels pertinents seront proposés aux visiteurs.

Le projet « Recto-verso " engagé dans le cadre de la coopération Rennes/ Nantes avec nos homologues nantais a permis la publication en ligne de deux nouveaux dossiers en ligne (Les petits papiers dans les archives et les visites présidentielles). 
PAYS DE LA LOIRE

Archives départementales de Loire-Atlantique

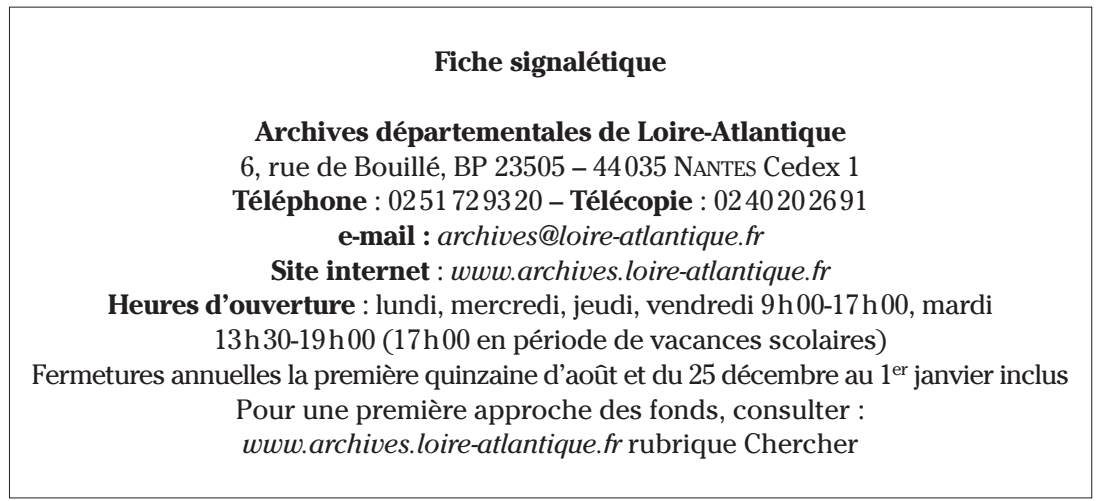

\section{1) Enrichissement des collections}

\section{Archives publiques}

Parmi les versements pris en charge en 2013 et 2014, on peut noter les ensembles suivants :

- Dossiers sur l'aéroport de Nantes Château-Bougon, remontant pour les plus anciens aux années 1960.

- Conseils d'administration de la caisse de compensation des allocations familiales du bâtiment et des travaux publics de l'ouest (depuis 1924) et de la caisse d'allocations familiales de Loire-Atlantique (à partir de 1946).

- Dossiers d'établissements d'enseignement privés du premier degré, remontant aux années 1930 .

- Dossiers sur le domaine routiers et les ouvrages d'art du XIX ${ }^{\mathrm{e}}$ siècle, provenant des anciennes subdivisions de l'Équipement.

- Registres de naturalisation depuis 1939.

- Archives de paroisses antérieures à la Révolution (complément à la série $\mathrm{G})$.

- Minutes des dernières études notariales n'ayant pas encore versé (Orvault, Héric, et Pontchâteau).

\section{Archives privées}

Parmi les fonds collectés, sont à signaler ceux :

- d'un tailleur de pierre et d'un ancien architecte en chef des monuments historiques, ayant travaillé tous les deux dans le département particulièrement intéressants pour l'histoire de l'architecture et des monuments; 
- du comité local du souvenir de Châteaubriant pour l'histoire de la seconde guerre mondiale et des commémorations;

- de La Guibourgère, château à Teillé

- d'Alain Chénard, ancien maire de Nantes et député

- du comité de réflexion de l'aménagement de l'université de Nantes lors de sa création

Comme nombre de services d'archives, celui de Loire-Atlantique a participé à l'opération nationale dite de la Grande Collecte autour du 11 novembre 2013. Une centaine de " petits fonds " ou pièces isolées sont venus ainsi complétés les archives déjà cosnervés sur le premier conflit mondial.

\section{Archives iconographiques et audiovisuelles}

Les retombées de l'exposition présentée en 2013 Dans l'œil du photographe ont permis l'entrée d'environ 1300 plaques de verre concernant non seulement le pays nantais mais aussi celui d'Ancenis (fonds de familles). L'exposition Design à bord présentée à partir du fonds de l'entreprise Mottais (emménageur de navires à Saint-Nicolas-de-Redon) a elle aussi suscité quelques dons et notamment celui de plans du paquebot Liberté réalisés par l'entreprise Lenglart dans les années 1950. A par ailleurs été acquis un album de photographies du France illustrant sa construction, son lancement et sa croisière inaugurale.

La convention avec la Cinémathèque de Bretagne a permis le collectage de films sur le carnaval de Nantes, la construction navale (lancement du Normandie, du Souverain des Mers), le monde industriel (charrues Huard de Châteaubriant, publicité la conserverie Cassegrain, confiserie nantaise Bonté), l'aéroport de Château-Bougon, les villégiatures à La Baule, l'institution pour sourds et malentendants de La Persagotière et les régates du cercle d'aviron nantais; et de films montrés au Caméra Club de Nantes.

\section{2) Instruments de recherche et publications}

L'actualité de la commémoration du centenaire de la guerre 1914-1918 a conduit à procéder au classement des archives relatives aux anciens combattants, aux prisonniers de guerre, évacués et réfugiés et aux comités spéciaux (service du contrôle de la presse de la XIe région militaire, comité de l'or et de l'emprunt de la défense nationale, de secours aux familles, etc.), et à la publication d'un guide de recherches pour reconstituer le parcours individuel des poilus. Un mémorial virtuel des soldats originaires de Loire-Atlantique morts au combat, dénommé " la Loire-Atlantique se souvient " a été mis en ligne en 2013. Il recense, sous la forme d'une base de données interrogeable (nom, lieux de naissance et de résidence, profession, unité d'affectation, date de décès...), plus de 27000 poilus, dont les notices peuvent être complétées par les internautes. 
Un important travail de conversion des répertoires sur fiches des minutes et répertoires notariaux de Nantes avant et après la Révolution, et de ceux des études hors Nantes postérieurs à la Révolution, a abouti à leur intégration dans le logiciel de gestion et d'interrogation des inventaires. Parmi les fonds dotés d'instruments de recherche, il faut signaler celui, privé, de la conserverie nantaise Saupiquet, et, celui, public, de la mission régionale créée avec la mise en place des circonscriptions d'action régionale en 1960, et mettant en lumière, jusqu'à la fin des années 1980, l'action de l'État en matière de développement économique et d'aménagement du territoire dans la région Pays de la Loire.

\section{3) Échos et nouvelles}

\section{Expositions}

- Cent ans de sports, 1850-1950, prolongation de l'exposition présentée depuis le 19 octobre 2012, jusqu'au 24 mars 2013.

- Dans l'œil du photographe, mettant en valeur trois fonds de photographes amateurs sur plaques de verre de la fin XIX ${ }^{\mathrm{e}}$-début Xx ${ }^{\mathrm{e}}$ siècle donnés aux Archives (11 septembre-20 décembre 2103). Les visiteurs de l'exposition ont été invités à identifier les vues qui ne l'avaient pas été lors de leur description.

- Design à bord, de septembre à décembre 2014, autour de l'emménagement intérieur des navires et paquebots

- La Loire-Inférieure dans la Grande Guerre, déclinaison itinérante de l'exposition C'était la guerre, présentée du 4 novembre 2014 au 12 avril 2015.

\section{Activités pédagogiques}

Les Archives proposent depuis 2012 des formations à destination des enseignants sur l'appropriation pédagogique des sources. Les thèmes abordés concernent les guerres mondiales, l'immigration, la société industrielle au XIX ${ }^{\mathrm{e}}$ siècle, le commerce triangulaire. Un nouvel atelier a été conçu "Pouvoir et féodalité au Moyen Âge ", de même qu'ont été publiés des fascicules en lien avec la guerre 14-18 ("Encadrer les consciences " et "Refuser la guerre : le pacifisme et ses manifestations durant la guerre", "L'expérience combattante " et "Les monuments aux morts").

\section{Fréquentation de la salle de lecture}

La tendance constatée les années passées de la baisse de fréquentation de la salle de lecture s'est confirmée en 2013 et 2014. Inversement proportionnelle à la montée en puissance de l'offre des Archives départementales en ligne, elle révèle l'évolution d'une société qui privilégie les services à distance, que corrobore les statistiques de fréquentation du site Internet. Celui-ci a été revu en 2014 (adresse, architecture et fonctionnalités), pour 
offrir une meilleure visibilité et permettant une meilleure accessibilité aux ressources en ligne.

\section{Numérisation}

La numérisation a porté, outre les compléments de fonds déjà traités, sur les rôles de désarmement des navires et ceux de l'impôt de la capitation du XVIIIe siècle, ainsi que le fonds des modèles et marques de fabrique du tribunal de commerce de Nantes. Ont aussi été numérisés les documents de la Grande collecte 14-18 représentant plus de 20000 pièces.

La politique d'océrisation de la presse a permis la constitution d'un corpus riche de 450000 pages portant sur 220 titres environ. Il est interrogeable en mode texte, et la pluralité des titres disponibles (information générale, politique, économique, presse de loisir, etc.) ainsi que la longue période couverte (1760-1940) permet d'intéresser tous les publics; chercheurs, généalogistes ou tout simplement curieux en histoire locale.

\section{Archives départementales de Maine-et-Loire}

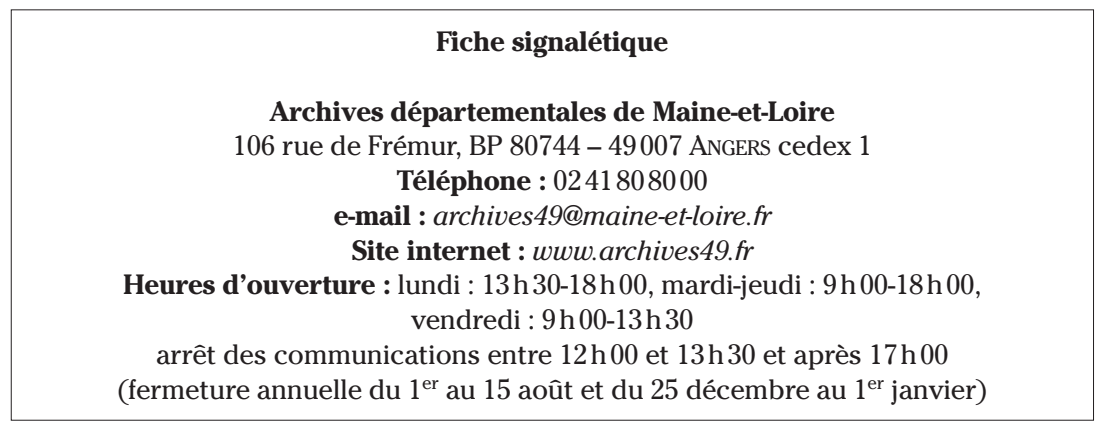

Les Archives départementales conservent 40 kilomètres de documents d'archives, couvrant une large période chronologique, du IX ${ }^{\mathrm{e}}$ siècle à nos jours. Les fonds médiévaux ecclésiastiques (abbayes de Fontevraud, de Saint-Florent...) sont particulièrement riches. Les fonds privés sont également très divers (familles, entreprises, associations...). La bibliothèque historique comprend plus de 15000 volumes et se présente comme un utile complément à la recherche. 


\section{1) Enrichissement des collections}

Plus de 1100 mètres linéaires de documents ont été collectés en 20132014, ainsi que près de 150 heures d'enregistrements sonores et 40 heures d'enregistrements audiovisuels.

En ce qui concerne les archives privées et iconographiques, l'opération de la Grande Collecte a suscité un écho sans précédent, incitant plus de 200 familles à faire connaître leurs souvenirs et écrits familiaux et à procéder parfois à des dons ou dépôts auprès des Archives départementales.

\section{Archives publiques}

Parmi les nombreux versements pris en charge, on peut signaler plus particulièrement les ensembles suivants :

- Sous-préfecture de Segré : secrétariat particulier, réglementation, affaires communales et intercommunales, contrôle de légalité (1966-2010).

- Conseil général : cabinet du président Christophe Béchu; direction de la culture et du patrimoine : subventions aux associations, affaires culturelles, suivi du patrimoine (1988-2012).

- Service de la publicité foncière de Segré, conservation des Hypothèques et service de l'Enregistrement (1876-1987).

- Service départemental des anciens combattants et victimes de guerre : cartes d'anciens combattants (guerres de 1939-1945, d'Indochine et de Corée) (1960-2009).

- Direction départementale de la cohésion sociale : développement des pratiques sportives, politique éducative territoriale, promotion des pratiques éducatives et citoyenneté des jeunes (1942-2005).

- Justice : tribunal d'instance de Cholet (1967-2008).

- Centre d'information et d'orientation d'Angers (1932-2012).

- Établissements hospitaliers : centre hospitalier de Cholet (1946-2007), hôpital Saint-Martin de Beaupréau (1844-2002).

- Centre national de danse contemporaine (1978-2005).

- Conseil d'architecture, d'urbanisme et de l'environnement (CAUE) (1978-2008).

- Anjou tourisme (comité départemental du tourisme de Maine-et-Loire) (années 1950-2013).

- Chambre départementale de métiers et de l'artisanat : fichier des artisants (1936-1987).

- Organismes sociaux : caisses d'allocations familiales d'Angers et de Cholet : administration générale, fonds de solidarité logement, action sociale... (1928-2012).

- Notaires : études de Vernoil-le-Fourrier, de Mûrs-Erigné.

- Archives communales déposées : Cuon, Le Lion-d'Angers. 


\section{Archives privées}

Quelques entrées en archives anciennes peuvent être notées :

- $349 \mathrm{~J}$ : chartrier du fief de Mazanger (1358-1873).

- $360 \mathrm{~J}$ : seigneurie de Beaupréau.

- 363 J : domaine du Plessis-Chivré (1337-1943).

- $365 \mathrm{~J}$ : domaine du Vau (XIV $-\mathrm{XV}^{\mathrm{e}}$ siècle).

Plusieurs associations importantes ont par ailleurs confié leurs fonds aux Archives départementales :

- $357 \mathrm{~J}$ : Fédération départementale Familles rurales.

- $358 \mathrm{~J}$ : Association départementale de lutte contre l'illettrisme (19862008).

$-366 \mathrm{~J}$ : SOS Femmes.

Le monde du travail est également représenté :

- $351 \mathrm{~J}$ : fonds de l'architecte Thomaseau.

- $352 \mathrm{~J}$ : CFDT-Métaux 49 Thomson.

- $353 \mathrm{~J}$ : Anjou Recherches Semences (ARES).

- $356 \mathrm{~J}$ : graineterie Jouffray de Chalonnes-sur-Loire.

\section{Archives iconographiques et audiovisuelles}

- Documents iconographiques : fonds photographique d'André Delluc, photographe à Beaufort-en-Vallée (43 Fi); vues des ateliers d'une manufacture de chaussures du Choletais (54 Fi); usine Thomson (63 Fi); École nationale des Arts et métiers d'Angers (64 Fi); atlas général du domaine de la Morosière et de ses dépendances (1817) (1 Fi).

L'opération Grande Collecte, en lien avec les commémorations de la Première Guerre mondiale, a également permis l'entrée de nombreux documents (photographies, dessins...).

- Archives sonores : ont été notamment débutées ou poursuivies les collectes de témoignages d'assistants sociaux (51 AV), de cyclistes (24 AV) et de combattants de la Seconde Guerre mondiale (50 AV).

\section{2) Instruments de recherche et publications}

\section{Inventaires}

Les classements d'archives effectués en 2013-2014 ont permis la mise à disposition de nombreux inventaires, parmi lesquels :

Archives modernes : 1924).

- $8 \mathrm{R}$ : fonds de la préfecture relatif à la Première Guerre mondiale (1914-

- 160 alpha : tribunal de commerce de Saumur (1798-1981).

- 183 alpha : service de la navigation (1828-1984).

Archives contemporaines :

- État des versements de l'Enregistrement (mise à jour). 
- $2269 \mathrm{~W}$ : SEITA (entrepôt des tabacs d'Angers, magasin de culture de Saumur, manufacture d'allumettes de Trélazé) (XIX siècle-1960).

- $2283 \mathrm{~W}$ : tribunal d'instance de Segré (1936-2007).

- 5 HS : hôpital de Cholet.

Archives privées :

- $338 \mathrm{~J}$ : chartrier de Fougeré (1330-1770).

Archives audiovisuelles :

- 1 AV 14 et 1 AV 15 : films fixes scolaires (1950-1970).

- $46 \mathrm{AV}$ : témoignages oraux relatifs aux jeunes filles et jeunes femmes des milieux ruraux des années 1930 aux années 1950.

Archives conservées dans les communes et ayant fait l'objet d'un classement et d'un inventaire par un archiviste contractuel :

- Brézé, Briollay, Brion, Cantenay-Epinard, Chartrené, Chazé-sur-Argos, Clefs, Corné, Coron, Durtal, Ecouflant, Gée, Huillé, La Boissière-sur-Evre, La Chaussaire, La Fosse-de-Tigné, La Jumellière, La Meignanne, La Ménitré, La Salle-de-Vihiers, La Séguinière, Le Fief-Sauvin, Le Lion-d'Angers, Le May-sur-Evre, Les Ponts-de-Cé, Longué-Jumelles, Louresse-Rochemenier, Melay, Montilliers, Montpollin, Montreuil-Bellay, Montreuil-sur-Loir, Montrevault, Passavant-sur-Layon, Pellouailles-les-Vignes, Pontigné, Saint-Aubin-de-Luigné, Saint-Clément-de-la-Place, Sainte-Christine, SaintGeorges-des-Gardes, Saint-Lambert-la-Potherie, Saint-Léger-des-Bois, Saint-Martin-d'Arcé, Saint-Pierre-Montlimart, Saint-Rémy-en-Mauges, Soulaines-sur-Aubance, Soulaire-et-Bourg, Souzay-Champigny, Tancoigné, Thorigné-d'Anjou, Varrains, Vihiers.

\section{Mise en ligne}

Le moteur de recherche mis à disposition sur le site internet pour la navigation dans les divers instruments de recherche s'enrichit progressivement au fil des opérations de classement de fonds ou de reprise d'anciens répertoires. Il compte actuellement plus de 220 inventaires accessibles en ligne.

\section{Publications}

À destination du public scolaire, quatre fiches pédagogiques ont été préparées : La construction des ponts sur la Loire et la Maine, Des hommes et des fruits, La conscription : deux siècles d'histoire (1798-1998), et Des Angevins au front pendant la Première Guerre mondiale.

\section{3) Échos et nouvelles}

\section{Salle de lecture}

Une réflexion sur l'ouverture et l'organisation du service de consultation a été menée en 2013. L'amplitude des horaires d'ouverture est passée de 
40,5 heures à 36 heures, par fermeture du vendredi après-midi. Cette demijournée est notamment l'occasion d'organiser à échéance régulière des ateliers de méthodologie de la recherche permettant de mieux appréhender les fonds des Archives départementales.

\section{Expositions}

L'action culturelle s'est organisée autour de deux thèmes d'exposition en 2013 : d'une part, une illustration de la carrière du maréchal de Bourmont, dont les archives conservées en mains privées ont fait l'objet, en 2012, d'une mesure de classement comme archives historiques; d'autre part, l'évocation de l'histoire de l'alimentation et de la gastronomie, illustrée d'exemples angevins, à travers l'exposition "La Table des Angevins ". Une exposition itinérante, " Résistances et Résistant(e)s en Anjou ", a aussi été mise au point en coopération avec l'association Résistance-Anjou.

L'année 2014 a quant à elle été dominée par la commémoration de la Première Guerre mondiale. Les Archives départementales ont proposé de septembre 2014 à février 2015 une exposition : " 1914. L'Anjou, de la paix à la guerre ", accompagnée de nombreux évènements et de la mise en place d'un site internet dédié à la Grande Guerre (www.grande-guerre-anjou.fr), destiné à être un support d'informations et d'initiatives pour porter la commémoration dans les prochaines années. L'opération a été clôturée par un ouvrage réunissant les apports des historiens et ceux de la Grande Collecte, paru en février 2015 : 1914-1918 : l’Anjou dans la Grande Guerre.

\section{Activités pédagogiques}

Près de 9400 élèves ont été accueillis en 2013-2014. Onze établissements scolaires ont par ailleurs participé en 2013 et 2014 au concours " Montrer l'histoire ", que les Archives départementales ont continué d'organiser.

Les Archives départementales s'impliquent également dans l'organisation du concours national de la Résistance et de la Déportation (organisation de la séance de lancement, accompagnement des enseignants et élèves, participation au jury, appui éditorial lors de la remise des prix). 


\section{Archives départementales de la Mayenne}

\begin{tabular}{|c|} 
Fiche signalétique \\
Archives départementales de la Mayenne \\
6 place des Archives, BP 80744 - 53000 LAVAL \\
Téléphone : 0243591090 - Télécopie : 02 435977 71 \\
e-mail : archives@lamayenne.fr \\
Site internet : www.archives53.fr \\
Heures d'ouverture : du lundi au vendredi de $9 \mathrm{~h} 00$ à $17 \mathrm{~h} 00$ \\
(fermeture annuelle la 1 re $^{\text {re }}$ quinzaine d'août)
\end{tabular}

\section{1) Enrichissement des collections}

\section{Archives publiques}

L'accroissement des collections a connu en 2013 et 2014 une hausse spectaculaire par rapport à l'année 2012 : il est ainsi entré au cours de ces deux années $630 \mathrm{~m}$ linéaires d'archives publiques définitives, parmi lesquelles il convient de signaler tout particulièrement :

- Les registres d'état civil de 1902 à 1937 versés par le greffe du tribunal de grande instance (54 m linéaires).

- Les dossiers de travaux sur les monuments historiques, accompagnés de nombreux plans, versés par le service territorial de l'architecture et du patrimoine (13 m linéaires)

- Un ensemble significatif de documents décisionnels versé par la chambre de commerce et d'industrie de la Mayenne (23 m linéaires)

Par ailleurs, plus de 76 mètres linéaires de minutes et répertoires de notaires sont entrés en 2014, ce qui constitue une très forte augmentation des fonds notariaux; ainsi ont été versés les minutes et répertoires des études de Saint-Pierre-des-Nids (1911-1924), Andouillé (1900-1938), La Baconnière (1907-1936), Chailland (1907-1938), Lassay (1700-1937), Martigné (1910-1938).

En ce qui concerne les dépôts d'archives communales, on peut souligner l'entrée des fonds de Niort-la-Fontaine (1611-1913) et de Lassay (17721920).

\section{Archives privées}

Parmi les entrées remarquables de 2013-2014, on peut signaler plus particulièrement les apports suivants :

- dépôt par ses propriétaires du chartrier de la Patrière en Courbeveille (XVI ${ }^{\mathrm{e}}$-XIX ${ }^{\mathrm{e}}$ siècle);

- archives des établissements Rivain, fabrique de chandelles, bougies et encaustique (1887-1970) - $532 \mathrm{~J}$; 
- fonds de Michel Denis, historien, professeur d'histoire contemporaine à l'université de Rennes 2, dont la thèse de $3^{\mathrm{e}}$ cycle publiée en 1967 s'intitulait L'Église et la République en Mayenne, 1896-1906, et la thèse de doctorat d'État, publiée en 1977, portait sur Les royalistes de la Mayenne et le monde moderne (XIX'-XXe siècle), $528 \mathrm{~J}(1970-2000)$.

- fonds de la Jeunesse ouvrière chrétienne de la Mayenne qui représente 6,20 m linéaires (1960-2000).

La Grande Collecte 14-18, commencée en 2013 et poursuivie en 2014, a été une grande réussite et a entraîné un essor considérable de la numérisation de complément, grâce aux dépôts et aux dons d'une cinquantaine de personnes. Parmi les acquisitions notables, il faut signaler notamment les fonds Vallet (1 100 clichés du 124 RI de Laval), et Ayrault de Saint Hénis (documents relatifs à l'hôpital temporaire ${ }^{\circ} 19$ de Laval).

\section{Archives iconographiques et audiovisuelles}

Les années 2013 et 2014 ont vu les collections iconographique s'enrichir d'un assez grand nombre de documents et fonds, parmi lesquels se distinguent plus particulièrement :

Des dessins :

- des dessins de Félix Désille, signés Liz : cour du Verger (auberge du pavillon, le beffroi Malhabri, le théâtre et le chemin de Saint-Julien, vers 1830); arrimage d'Hydouze (rue de la Rivière vers 1810) - 47 Fi 74-77;

- un dessin au lavis de Berthe Marcou représentant la cour du château de Laval - 3 Fi (1950-1990);

- le fonds Jean Lesourd comprenant des carnets de croquis, des dessins, des aquarelles, des huiles sur toiles, des livres - $52 \mathrm{Fi}$ (XIX ${ }^{\mathrm{e}}-\mathrm{XX}$ e siècles);

- un ensemble de dessins relatifs à la restauration de l'église de SaintDenis d'Anjou signés par l'architecte Gendron - 53 Fi (1861-1861);

- un ensemble de dessins, calques, plans, photographies relatifs à la restauration de la cathédrale de Laval. Un ouragan avait fortement endommagé le monument en 1702. Les travaux furent entrepris à partir de 1885, menés par Jean-Camille Formigé.

Des photographies isolées ou regroupées en album :

- photographies relatives à l'industrie lavalloise (usines sur les rives droite et gauche de la Mayenne, XIX siècle) - 10 Fi 134-143;

- un album de quatre photographies relatives à la fête du Genest, août 1926 - 11 Fi (1926-1926);

- une photographie de classe de l'école de Montsûrs en 1900 - 11 Fi (1900-1900);

- quatre photographies relatives à une caserne de soldats allemands établie à Laval, avec prisonniers de guerre français - 11 Fi (1939-1945);

- l'album des Arcis : 23 photographies relatives à Soulgé-le-Bruant (château des Arcis, foire,) et au viaduc de Laval - 50 Fi (1890); 
- plusieurs albums de photographies relatives à la ville de Mayenne au début du $\mathrm{xx}^{\mathrm{e}}$ siècle (ponts, rivière, etc.) et à la famille de la Charie : Châtelain, Saint-Denis-de-Gastines - 51 Fi (1900-1920).

\section{2) Instruments de recherche et publications}

L'année 2013 a vu l'achèvement des classements de fonds très demandés par les chercheurs : fonds Francis Robin (seconde guerre mondiale), fonds Félix Désille (carnets de dessins et notes sur le département), fonds Auguste Alleaume (maître-verrier).

Les registres d'état civil de 1902 à 1937 versés en 2013 ont été classés et sont à la disposition du public dans la salle de lecture depuis février 2014 .

Il faut ajouter à cela les publications sur Internet de plusieurs instruments de recherche (concernant notamment les archives communales).

\section{3) Échos et nouvelles}

L'année 2013 a été marquée par plusieurs événements culturels et médiatiques.

La plaque symbolisant le label "Patrimoine $d u X X^{e}$ siècle ", attribué au bâtiment des Archives, a été inaugurée à la veille des Journées européennes du patrimoine par Jean Arthuis, président du conseil général, en présence de Dominique Perrault et Alfred Heude, architectes. Le même jour a eu lieu le vernissage de l'exposition " Place des Archives ", présentée de septembre 2013 à mars 2014. Elle a connu un vif succès.

Par ailleurs, les Journées européennes du patrimoine ont réuni 520 personnes aux Archives départementales, qui toutes ont visité l'exposition en plus de la traditionnelle découverte du bâtiment et des collections.

Au cours de l'année, environ 150 autres personnes ont pu bénéficier d'une visite commentée des Archives départementales et/ou de l'exposition "Place des Archives".

Enfin, les Archives départementales ont proposé au cours de l'année deux conférences :

- l'une de Rémy Foucault sur «La persécution antisémite dans le département de 1940 à 1944 » accompagnée du témoignage de Joseph Weismann, enfant juif caché en Sarthe pendant la seconde guerre mondiale, auteur d'Après la rafle (Michel Laffon, 2011), qui a inspiré le film La Rafle de Roselyne Bosch. Cette manifestation a réuni 70 personnes;

- l'autre de Jean-Louis Beaucarnot, spécialiste français de la généalogie, venu présenter et commenter le hors-série conçu et réalisé par Ouest-France et La Revue française de généalogie avec la collaboration des Archives départementales "La généalogie en Mayenne ". Cette conférence a été suivie par 110 personnes.

Les Archives départementales ont également participé à plusieurs émissions radiophoniques sur France Bleu Mayenne, le 15 septembre au sujet de l'exposition " Place des Archives " et du label "Patrimoine du XX siècle ", 
et le 29 novembre pour un sujet sur les documents publicitaires conservés aux Archives départementales.

Par ailleurs, un reportage télévisé relatif à une affaire criminelle de la fin du XIX siècle (affaire de l'abbé Bruneau), en partie réalisé aux Archives à partir des documents de procédure, des registres d'écrou et des journaux de l'époque, a été diffusé par France 3 Télévision le 6 juin et le 17 octobre 2013.

Enfin, Pierre Guicheney, écrivain et réalisateur, a tourné plusieurs scènes d'un reportage sur le textile en Mayenne aux Archives départementales.

L'année 2014, quant à elle, a été aussi importante pour la valorisation des fonds d'archives. Elle a été marquée notamment par le coup d'envoi du centenaire de la guerre de 1914-1918.

Deux expositions ont été montées et présentées par le service :

- "Alleaume : lumière sur une famille d'artistes".

D'origine angevine, la famille Alleaume a donné à la Mayenne deux acteurs de la scène artistique locale de la première moitié du xx siècle : Auguste et Ludovic Alleaume. Leur père, Auguste Symphorien, horloger et créateur, est le concepteur de l'horloge astronomique du Musée des sciences de Laval. De 1893 à 1937, Auguste Alleaume, peintre verrier, a créé les vitraux de nombreuses églises de la Mayenne. Son frère Ludovic, artiste peintre installé à Paris, l'a secondé dans la réalisation des vitraux et a participé à la décoration de plusieurs édifices en Mayenne.

Cette exposition, présentée du 28 avril au 29 août, a fait partie d'une opération plus vaste autour de la famille Alleaume. Elle a permis de valoriser le fonds d'archives familiales donné aux Archives départementales en 2008.Le Centre d'interprétation de l'architecture et du patrimoine de SainteSuzanne a réalisé de son côté une exposition sur l'atelier du maître-verrier. Enfin, le musée du Vieux-Château de Laval a présenté en fin d'année une rétrospective de l'œuvre de Ludovic.

- "Moissons rouges : un Mayennais dans la Grande Guerre ».

S'inscrivant dans la commémoration du Centenaire de la guerre de 1914-1918, cette exposition évoque les conditions de vie des soldats sur le front à travers le récit de l'expérience d'un Mayennais, Albert Filoche, né à Grazay, brancardier au 124e régiment d'infanterie. Conservées précieusement au sein de sa famille, les archives d'Albert Filoche forment un ensemble exceptionnel constitué de lettres et de carnets journaliers, de poèmes et de récits. L'exposition présentée a été conçue à partir d'une première exposition réalisée en 2005 avec la collaboration de l'historienne Jocelyne Dloussky. Remaniée et enrichie grâce aux documents collectés depuis cette date, elle permet aussi de mettre en lumière la participation exceptionnelle des Mayennais à la Grande Collecte.

L'exposition, présentée du 20 septembre 2014 au 30 janvier 2015, a été accompagnée d'un programme d'animations variées qui ont connu un vif succès : trois conférences ont été proposées : Albert Filoche, un poilu sur 
le front de Jocelyne Dloussky, historienne, Retrouvez vos ancêtres en 14-18 de Pierrick Bourgault, journaliste indépendant et photographe, Du front à l'Asile de Hervé Guillemain et Stéphane Tison, maîtres de conférences à l'université du Maine; deux concerts de la compagnie Ellébore, "Chansons pour une ville en guerre " ont fait salle comble avec 110 personnes à chaque représentation; deux projections du court métrage Sur la trace des mutins, évoquant les soldats mutins de 1917 envoyés aux travaux forcés dans les carrières de Voutré ont réuni 90 élèves lors d'une séance scolaire et 45 personnes en soirée; elles ont été suivies d'une rencontre avec le réalisateur Jean-Marc Allaine, le producteur Antoine Glémain et l'historien Jacques Cousin.

\section{Site Internet}

En 2014, le nombre d'images consultées sur le site des Archives départementales a dépassé les 29 millions. (26 millions en 2012).

\section{Numérisation}

Les travaux de numérisation ont porté en 2013 sur les registres des états de section du cadastre napoléonien, des listes nominatives de recensement de population (1946-1975) et des microfilms, en 2014 sur les registres d'état civil de 1902 à 1937 versés en 2013.

En interne, au cours de ces deux années, les opérations de numérisation ont concerné essentiellement les documents collectés à l'occasion de la préparation des commémorations du centenaire de la Première Guerre mondiale.

\section{Archives départementales de la Sarthe}

\section{Fiche signalétique}

Archives départementales de la Sarthe

9 rue Christian-Pineau - 72100 LE MANS

Téléphone : 0243547474 - Télécopie : 0243547446

e-mail : archives@sarthe.com

Site internet : $w w w$.archives; sarthe.fr

Heures d'ouverture : lundi au jeudi, 13h00-17h 30 ; vendredi, 8h 30-17 h 30;

un samedi tous les mois et demi, $8 \mathrm{~h} 30-13 \mathrm{~h} 00$

(fermeture annuelle la $1^{\text {re }}$ semaine de septembre et du 25 décembre au $1^{\mathrm{er}}$ janvier) 


\section{1) Enrichissement des collections}

\section{Archives publiques}

Dans le contexte du regroupement des services de l'État sur le site de l'ancienne caserne Paixhans, les Archives départementales ont accueilli 913 mètres linéaires de documents en 2013 et 1438 mètres linéaires en 2014.

Parmi ces versements, on relève plus particulièrement :

- une riche série sur les ouvrages hydrauliques remontant au XIX ${ }^{\mathrm{e}}$ siècle (versement de la direction départementale des Territoires);

- les plans parcellaires et dossiers de gestion des forêts domaniales de Bercé et de Perseigne au XIX ${ }^{\mathrm{e}}$ siècle (versement de l'office national des Forêts);

- les archives administratives du Centre hospitalier général du Mans, des années 1850 à 1970 ;

- les dossiers de la cour d'assises de la Sarthe depuis 1935.

Au titre des dépôts d'archives communales, on signalera le fonds ancien très complet de la commune de Mayet, chef-lieu de canton d'envergure, et de son comice agricole (34 mètres linéaires).

\section{Archives privées}

Plusieurs entrées remarquables demandent à être signalées :

- les dossiers de travail et de documentation de l'historien Michel Rosier, spécialiste de l'histoire politique de la Sarthe sous les IV et Ve Républiques;

- les archives du syndicat CGT Renault Le Mans de 1947 à 2005;

- le fonds de l'association Culture et liberté en Sarthe qui retracent trente années d'engagement associatif dans le domaine de l'insertion;

- les archives d'activité de Pierre-Jean-Baptiste Lelong, lieutenant général de la sénéchaussée puis procureur syndic du district de Château-du-Loir (1770-1794), auxquelles étaient réunis les cahiers de plaintes et doléances des trente-neuf paroisses du ressort en 1789;

- les archives personnelles du docteur Rémi Charles, président du Groupement d'étude des maladies en Sarthe, principalement relatives à la mise en place du dépistage du cancer de sein en Sarthe (1986-2013);

- cahiers de notes, manuscrits, travaux et ouvrages de Paul Vallin, notamment relatifs à la Nouvelle Calédonie et à l'outre-mer français (années 1980);

- les archives de l'association de gestion du Centre socioculturel Simone Signoret de Mulsanne (1980-2010);

- le fonds de l'association Croyants en liberté Sarthe (1993-2008);

- les dossiers du pré-inventaire réalisés par l'association Sablé et alentours;

- les archives de l'Association sarthoise pour l'érection d'un mémorial aux combattants d'Algérie, de 1992 à 2007 (ASEMACA); 
- près de 14000 plans " constructeur " sur calque, produits par les entreprises Autobus Verney - SAMV - CBM Le Mans (1954-1986).

Le chartrier du Château de Courtanvaux (XIVe-Xx siècle), regroupant des archives personnelles, familiales, seigneuriales et de gestion domaniale des familles de Souvré, Le Tellier/Louvois et de Montesquiou, a fait l'objet d'un dépôt sous contrat par la commune de Bessé-sur-Braye.

Le Conseil général a fait l'acquisition en 2013-2014 des fonds suivants :

- des archives personnelles, dossiers de travail, correspondance érudite et mondaine, almanachs, cartes postales et documentation de l'abbé Bidault, spécialiste des étains religieux des XVII et XVIII ${ }^{\mathrm{e}}$ siècles, relatifs aux arts et traditions populaires, à des manifestations et cérémonies et au clergé diocésain pendant la Seconde Guerre mondiale (1882-1969);

- un ensemble d'archives seigneuriales et domaniales intéressant la région de Saint-Calais du XVII ${ }^{\mathrm{e}}$ au XIX ${ }^{\mathrm{e}}$ siècle;

- des aquarelles, lithographies, estampes d'artistes de renom (Gamy, Rob Roy, Géo Ham) ayant trait au Grand prix de France et aux 24 heures du Mans;

- une collection produite par un photographe professionnel, composée de 176 ektachromes de vues aériennes de la Sarthe, prises de 1981 à 1987.

Par ailleurs, des témoignages oraux ont été collectés dans le cadre du programme "Sauvegarde de la parole sarthoise " sur la vigne et la tonnellerie et sur le monde ferroviaire en Sarthe qui ont permis la réalisation, en partenariat avec le service Archives et documentation de la SNCF, de trois films documentaires courts présentés dans le cadre de l'exposition L'aventure du rail en Sarthe, des origines à la régionalisation.

\section{2) Instruments de recherche}

37 instruments de recherche analytiques et inventaires synthétiques ont été rédigés au cours des deux années, dont le répertoire numérique détaillé du fonds Dorizon-Compagnie des tramways de la Sarthe.

Dans le cadre d'un stage de l'Institut national du patrimoine a été réalisé le répertoire numérique détaillé du fonds de la section commerciale du tribunal de première instance de La Flèche.

\section{3) Échos et nouvelles}

\section{Expositions}

En 2013, la manifestation consacrée à L'aventure du rail en Sarthe, conçue autour d'une exposition avec catalogue, présentée à l'abbaye de l'Épau du 5 juillet au 15 septembre, et de déclinaisons itinérantes sur les sites des associations partenaires, a connu une excellente fréquentation. 8120 personnes au total ont découvert l'exposition elle-même, participé aux visites de sites, aux projections de films ou aux journées d'étude (L'Épau, 13 septembre) et d'animation (Château-du-Loir et Montabon, 12 octobre). 
Cette manifestation a été l'occasion pour les Archives départementales, les Archives historiques de la SNCF et les cinq associations partenaires de décliner une politique d'animation territoriale destinée à un large public, tout au long de l'été et de l'automne 2013, avec un bon relais médiatique (de la part de France Bleu Maine en particulier). La notoriété des Archives départementales s'en est trouvée renforcée et la fréquentation du site de l'abbaye de L'Épau dynamisée.

Une déclinaison " tonnellerie " de l'exposition itinérante Des vignes, des vins, des hommes (conçue en 2012) a été présentée avec grand succès à l'espace Carnuta, à Jupilles, de mai à septembre 2013, où sont passés plus de 4200 visiteurs. Cette exposition a également connu une fréquentation remarquable à la cave Lelais, à Lhomme, où 1000 visiteurs se sont pressés sur un unique week-end.

Dans le cadre des célébrations nationales, le 11 avril 2013, une exposition et une conférence de Jean-Yves Le Naour ont été organisées à la Maison du Département à Mamers, en hommage à la grande figure politique que fut Joseph Caillaux, né au Mans le 31 mars 1863.

En 2014, l'exposition labellisée commémorative du centenaire de la première guerre mondiale Des étrangers en Sarthe, des Sarthois à l'étranger a été présentée simultanément dans l'espace d'exposition des Archives départementales et à l'abbaye de l'Épau (version itinérante sur panneaux), du 5 juillet au 17 septembre, ainsi que du 7 au 13 septembre dans le dortoir de l'abbaye. Sa fréquentation globale est de l'ordre de 1300 visiteurs. Elle a, par ailleurs, été présentée au château de Sablé-sur-Sarthe (centre Joël Le Theule de la Bibliothèque nationale de France) et, sous forme itinérante, à la Maison du département à Mamers et à l'espace culturel de Saint-Cosmeen-Vairais. Elle a donné lieu à l'impression d'un catalogue de 69 pages, produit à 1000 exemplaires.

Fruit d'un partenariat original avec la délégation de la Sarthe des Amis de la Fondation pour la mémoire de la Déportation, le Musée mémorial de Dora (Allemagne, Niedersachsen) avec la participation du lycée du Mans sud, les expositions conçues autour des dessins retrouvés du camp de déportés de Holzen et la vie de leur auteur Camille Delétang, sous le titre Redécouverts - Camille Delétang, un homme dans son siècle de Verdun à la Résistance, présentée dans l'espace d'accueil des Archives du 9 octobre 2014 à la mi-février 2015, ont connu un beau succès, grâce à une très forte implication associative (visites commentées quasi quotidiennes, relais auprès des médias...), puisqu'elle a accueilli 1016 visiteurs en l'espace de deux mois et demi seulement.

La circulation de quatre expositions itinérantes conçues par les Archives au cours des années antérieures - tout particulièrement celle de 2012 sur la vigne - a connu un très grand succès public dans le territoire départemental (1660 visiteurs), tout particulièrement dans le terroir viticole de Ruillé-sur-Loir, où 900 personnes se sont déplacées en l'espace de 12 jours. 
À l'occasion des Journées européennes du Patrimoine 2013, les visites commentées de l'exposition temporaire sur L'aventure du rail en Sarthe ont drainé 1700 visiteurs entre l'abbaye de l'Épau (samedi et dimanche) et les sites partenaires du milieu associatif (Beillé, siège de l'association Transvap et Semur-en-Vallon, siège du Petit train de Semur-en-Vallon).

Dans une configuration plus classique, en 2014, 40 visiteurs ont pris part à une visite-conférence autour de l'exposition du centenaire de la première guerre mondiale " Des étrangers en Sarthe, des Sarthois à l'étranger " (samedi après-midi) et 198 personnes ont été accueillies aux Archives départementales à l'occasion des Journées européennes du Patrimoine (dimanche après-midi).

\section{Fréquentation de la salle de lecture}

1006 lecteurs ont été accueillis en 2013 (999 en 2014), à l'occasion de 3222 séances de travail (3058 en 2014), soit 14431 documents communiqués (12373 en 2014). $51 \%$ des visiteurs ont effectué des recherches généalogiques (48 \% en 2014) et $23 \%$ des recherches à caractère scientifique (35\% en 2014).

\section{Activités pédagogiques}

1239 élèves ont été accueillis par le service éducatif sur l'année scolaire 2013 (1 403 en 2014), dont 500 élèves de cours élémentaire (307 en 2014) et 628 collégiens (753 en 2014), auxquels s'ajoutent élèves reçus dans le cadre de visites d'expositions. Par ailleurs, 50 étudiants (60 en 2014) ont pris part à des travaux encadrés aux Archives (notamment en licence 3 d'histoire) et 33 personnes handicapées ont également bénéficié d'un accueil dédié dans le cadre des visites de groupe sur mesure.

Comme les années antérieures, les Archives départementales ont organisé, à l'initiative de l'Inspection académique et en partenariat avec l'ONAC et les associations d'anciens combattants, une réunion de mobilisation du monde enseignant autour du concours national de la Résistance et de la Déportation en novembre 2013 et décembre 2014.

\section{Projets scientifiques}

À l'initiative des Archives départementales, le groupe de travail associant des enseignants-chercheurs, des étudiants de l'Université du Maine, les présidents et des membres des sociétés d'histoire et d'archéologie, les Archives départementales de la Sarthe et les Archives municipales du Mans, a activement poursuivi ses travaux en vue d'assurer l'édition scientifique et la publication en ligne des délibérations des conseils de ville du Haut-Maine antérieures à la Révolution (Beaumont-le-Vicomte, Châteaudu-Loir, Fresnay, La Ferté-Bernard, La Flèche, Le Mans, Mamers, Sablé, SaintCalais, Sillé-le-Guillaume). 


\section{Numérisation}

En raison de leur fort intérêt pour de nombreux aspects de l'histoire locale, les originaux et microfilms des répertoires de notaires de la Sarthe (XVII ${ }^{\mathrm{e}} \mathrm{XIX}{ }^{\mathrm{e}}$ siècle) ont été numérisés par sous-traitance en 2013 en vue de leur mise en ligne ultérieure (102000 images).

Dans la même optique, ce sont les registres des tables de successions et absences de l'Enregistrement qui ont été numérisés en 2014 (184062 vues produites), en vue d'une mise en ligne en 2015.

En interne, en 2013, 12068 vues numériques ont été réalisées dans le cadre de la grande collecte 1914-1918 (250 opérations), de la numérisation du chartrier de la Bouillerie et des activités du service éducatif ou de la préparation des expositions.

En interne toujours a été poursuivie en 2014 la numérisation du fonds d'archives seigneuriales et domaniales du château de La Bouillerie à Crosmières, $\mathrm{XVI}^{\mathrm{e}}$-XIX ${ }^{\mathrm{e}}$ siècle (5850 vues produites).

\section{Diffusion en ligne}

L'indexation collaborative à l'année est désormais possible sur les registres numérisés des registres paroissiaux et de l'état civil.

Par ailleurs, dans le contexte du centenaire de la déclaration de guerre, ont été mis en ligne, en juillet 2014, les registres matricules militaires des classes de conscription de 1879 à 1922 .

\section{Archives municipales d'Angers}

\begin{tabular}{|c|}
\hline Fiche signalétique \\
Archives municipales d'Angers \\
Service Archives-Documentation-Photothèque de la ville d'Angers \\
Hôtel de Ville, boulevard de la Résistance-et-de-la-Déportation, \\
CS 80011 - 49020 AnGERs cedex 02 \\
Téléphone : 0241054281 - Télécopie : 0241053911 \\
e-mail : gilles.neau@ville-angers.fr (secrétariat) \\
sylvain.bertoldi@ville-angers.fr (direction) \\
Site internet : www.angers.fr/archives \\
Heures d'ouverture : du lundi au vendredi de 14h00 à $17 \mathrm{~h} 00$ \\
(fermeture annuelle la 1 ${ }^{\text {re }}$ quinzaine d'août) \\
Pour une première approche des fonds consulter \\
le site internet rubrique Archives municipales \\
\hline
\end{tabular}




\section{1) Enrichissement des collections}

\section{Archives publiques}

De nombreux audits d'archives ont été menés à la faveur de déménagements ou réorganisations de services. 330 mètres linéaires ont été collectés, parmi lesquels il faut signaler :

- projet et contrat éducatif local (2007-2010);

- menus des réceptions officielles organisées par la Ville (1990-2008);

- charte culture et solidarité, ateliers Gaubourgs, Tours de scènes (19982008);

- Bâtiments municipaux : organisation de la direction, gestion, travaux (1938-1990);

- centre nautique du lac de Maine, création et gestion (1978-2011);

- relations internationales : partenariat Angers-Bamako (1972-2006), dossiers des ambassadeurs des villes jumelles (1986-2013);

- opérations d'urbanisme, d'aménagement et renouvellement urbain (19692014);

- équipements culturels (1970-2010);

- projet Rives nouvelles (2002-2014);

- politique de la ville, associations, jeunesse (quartiers Monplaisir - GrandPigeon) (2002-2010);

- dossiers suivis par la direction générale des services (1982-2013).

\section{Archives privées}

Parmi les 133 mètres collectés figurent de beaux ensembles :

- archives de l'entreprise Thomson (années 1960-début Xxi ${ }^{\mathrm{e}}$ siècle);

- syndicat de gestion de l'île Saint-Aubin, gestion du territoire (18252012);

- groupement musical Fanfare du IVe arrondissement (1891-1991);

- association Présence de l'art contemporain Angers (PACA) (19852013);

- archives familiales Belliard-Poirier, Chaslot, Jubin-Defoix;

- épicerie Huchet, rue Toussaint, Angers (gestion, fournisseurs) (1938, 1949);

- section d'Angers de la lutte nationale contre le taudis (vers 1920-1945);

- archives personnelles de Christine Brisset, fondatrice des Castors Angevins, concernant le logement social à Angers (années 1940-1960);

- université angevine du temps libre (UATL) (1974-2013);

- compagnie Jo Bithume (spectacle des arts de la rue, fondatrice des Accroche-Cœurs) (1982-2014). 


\section{Archives iconographiques et audiovisuelles}

32170 nouvelles pièces sont entrées dans les fonds iconographiques, patrimoniaux et de communication : versements des différents photographes qui travaillent pour la Ville, dons et achats, numérisation de fonds privés...

Il faut noter en particulier :

- plaques de verres de la Société anonyme des filatures, corderies et tissages Bessonneau;

- albums de souvenirs et de voyages d'André Godin, commerçant et artiste amateur (1894-1940);

- clichés des expositions organisées par Présence de l'art contemporain Angers;

- abattoirs d'Angers, dessins d'Auguste Ventrou (1910-1914).

Des documents de collections privées ont été numérisés pour enrichir les fonds, notamment une sélection de clichés sur le footballeur Georges Bykadoroff et les clichés du livre Angers mémoire pris en 1997 par Éric Jabol.

L'ensemble des plaques de verre conservées aux Archives a fait l'objet d'une opération de restauration.

\section{Objets}

Médailles ayant appartenu au maire Jean Turc (1963-1977).

\section{2) Instruments de recherche et publications}

\section{Instruments de recherche}

En plus des classements ouverts en continu pour les séries d'archives qui s'accroissent au jour le jour (versements administratifs contemporains, archives privées, documents iconographiques), les inventaires suivants ont été rédigés :

- police - Hygiène et sécurité publiques (2 et 7 I);

- bâtiments municipaux (52-126 M);

- association syndicale des propriétaires de l'île Saint-Aubin (73 J);

- association Présence de l'art contemporain Angers (76 J);

- université angevine du temps libre (87 J).

\section{Autres publications}

"Angers depuis 1945 ", dans Guide Angers Ville d'Art et d'Histoire, 2013.

"Banquets institutionnels à Angers sous l'Ancien Régime ", dans Archives d'Anjou, 2013.

"Banquets institutionnels et repas officiels à Angers de 1790 à 2010 ", dans Archives d'Anjou, 2014.

"Nouvelles acquisitions des archives municipales d'Angers ", dans Archives d'Anjou, 2014. 
Chronique historique mensuelle dans Vivre à Angers. Le texte intégral, plus développé, est en ligne sur internet à l'adresse indiquée ci-dessus.

\section{3) Échos et nouvelles}

\section{Fréquentation de la salle de lecture - nombre de documents communiqués}

312 lecteurs en 2013, 339 en 2014 ont consulté chaque année environ 3000 documents. L'ensemble des communications, comprenant celles effectuées au profit des services de la mairie et les clichés numériques envoyés par le service Photothèque, s'élève à 11420 documents pour 2013, 8121 en 2014.

Une version test du site "Archives en ligne " permettant des recherches transversales dans un grand nombre de fonds a été ouverte en salle de lecture.

\section{Activités pédagogiques}

Des cours de paléographie sont donnés à l'université et, pour tous les passionnés de recherches, dans le cadre de l'Association des Amis des Archives de l'Anjou (4 A).

Un travail de recherche et de constitution de bases de données sur les commerces angevins ( $\mathrm{XVIII}^{\mathrm{e}}-\mathrm{XX} \mathrm{e}^{\mathrm{e}}$ siècles) a été mis en place avec un groupe de chercheurs bénévoles autour de Sylvette Robson.

\section{Numérisation}

Les fonds suivants ont fait l'objet d'une campagne de numérisation : cahiers de doléances, comptes anciens (1367-1712), registres de la capitation (1712-1789), arrêtés municipaux (1793-1919), états de sections du cadastre (1810 et 1840), grand cartulaire analysé de la ville, registres de la contribution mobilière (1791-1792), délibérations du conseil municipal (2007-2014), affiches. La numérisation des recensements de la population, déjà entamée il y a quelques années, a été complétée (1790-1836), de même que celle des listes électorales (1796-1889). 


\section{Archives municipales de Laval}

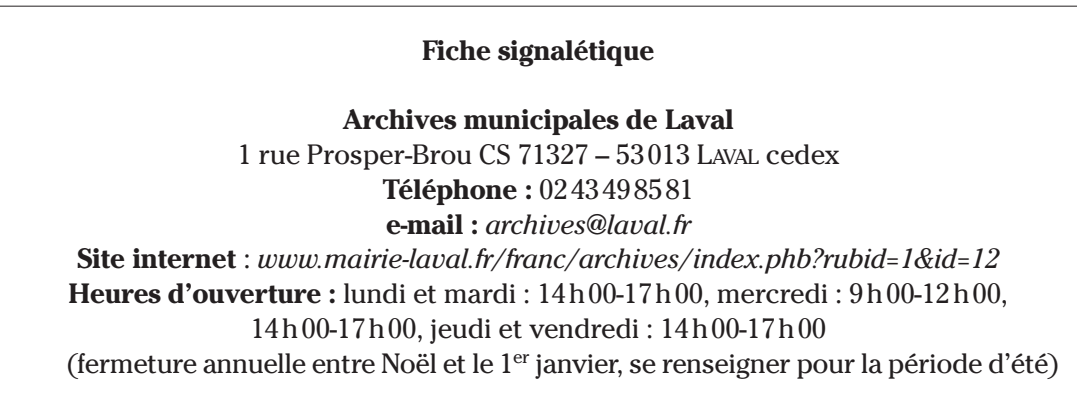

Une bonne partie du fonds moderne antérieur à 1950 est encore déposée aux Archives départementales de la Mayenne.

\section{1) Enrichissement des collections}

\section{Archives publiques}

L'accroissement net total a été de $67 \mathrm{~m}$ linéaires au cours des années 2013 et 2014.

Une nouvelle convention a été signée avec Laval-Agglomération pour la gestion des archives de l'intercommunalité

\section{Archives privées}

Parmi les dons et dépôts reçus en 2013 et 2014 on notera la licence de pilote et le carnet de vol de la première femme pilote de l'aéro-club de la Mayenne, des archives du comité de jumelage Laval-Boston (en cours de classement) et 100 photographies du Carnaval prises entre 1994 et 2002 (essentiellement des portraits).

\section{2) Instruments de recherche}

Le classement des archives de l'intercommunalité a été poursuivi. Il concerne pour l'instant les archives du District.

Le classement des versements a été poursuivi. En particulier ont été classés les dossiers de lotissement.

Des inventaires papier ainsi qu'un poste de consultation du module web sont mis à la disposition des lecteurs. 


\section{3) Échos et nouvelles}

\section{Salle de lecture}

La fréquentation du service a notablement augmenté mais elle reste modeste.

\section{Valorisation}

Les Archives municipales ont participé à l'opération « Industrielle attitude " permettant au grand public d'accéder aux entreprises mayennaises et aux Journées du Patrimoine. À ces occasions, une visite du service a été proposée ainsi qu'une mini-exposition de documents remarquables.

La responsable du service est intervenue lors des "Cafés d'histoire ", animation d'été proposée par le service Patrimoine : une causerie d'une demi-heure autour d'un café.

\section{Numérisation}

Ont été numérisés des documents du fonds Trouillet (1943-1955), des diapositives des années 1970 ainsi que des diapositives prêtées pour reproduction et concernant un voyage à Boston (Angleterre) dans le cadre du jumelage.

\section{Archives Ville Le Mans et Le Mans Métropole}

\begin{tabular}{|c|}
\hline Fiche signalétique \\
Archives municipales et du Mans Métropole, communauté urbaine \\
102, rue Gambetta -72039 Le Mans cedex 9 \\
Téléphone : 0243474903 - Télécopie : 0243474368 \\
e-mail : archives@ville-lemans.fr \\
Heures d'ouverture : lundi-vendredi, $8 \mathrm{~h} 30-12 \mathrm{~h} 00$ et $13 \mathrm{~h} 00-17 \mathrm{~h} 00$ \\
(fermeture des portes 15 minutes avant)
\end{tabular}

\section{1) Enrichissement des collections}

\section{Archives publiques}

Parmi les versements pris en charge en 2013-2014, on peut noter les ensembles suivants : 
- aménagement urbain : circulation urbaine (1984-2011), transport urbain (2002-2012), zones d'aménagement (1997-2010), opération d'urbanisme (1986-2008), plan d'aménagement urbain (1994-2008);

- assemblées : conseil municipal (2010-2011), municipalité (2011-2012), conseil communautaire (2010-2011), bureau communautaire (2011-2012);

- budget et fiscalité : budget Ville et Le Mans Métropole (2013-2014);

- espaces verts : aménagement des aires de jeux et des espaces verts (1974-2011);

- population : registre des naissances (1913), registre des mariages (1937), registre des décès (1937), tables décennales naissances (1903-1912);

- services généraux : arrêtés du Maire (2012-2013), arrêtés du Président de Le Mans Métropole (2012-2013), autorisation d'ouverture exceptionnelle de magasins et liquidation de marchandises (2007-2008);

- théâtre municipal : programmation culturelle (1999-2009);

- urbanisme : permis de construire de la ville du Mans et communes de Le Mans Métropole (2005-2008), plan d'occupation des sols (1987-2008).

\section{2) Instruments de recherche et publications}

- 7859 notices ont été saisies et indexées sur le logiciel de gestion d'archives;

- 13851 notices ont été saisies et indexées sur le logiciel de gestion d'archives Avenio qui fait office d'instrument de recherche.

- Mise à jour des répertoires numériques existants pour les séries modernes.

\section{3) Échos et nouvelles}

\section{Numérisation}

20786 fichiers numériques ont été produits. Ils concernent : 4 registres de tables décennales naissances (1762-1791, 1903-1912), 1 registre de tables décennales mariages (1782-1791), 1 registre de tables décennales décès (1752-1761), 4 registres naissances (1911-1912), 5 registres mariages (19331937), 5 registres décès (1933-1937), 24 registres de délibérations municipales (1943-1967), une sélection de 274 plans de permis de construire (1956-1961), 3592 reportages photographiques architecture et opération d'urbanisme (1968-1972), 108 cartes postales (XIX $-\mathrm{XXI}^{\mathrm{e}}$ siècles).

\section{Restauration}

Poursuite de la campagne de restauration : 25 listes électorales (19231932), 16 registres paroissiaux (1584-1791), 8 registres d'état civil (1793-1863), 1 registre du bureau de bienfaisance (1835-1863), 6 registres de recensement pour le logement militaire (1871-1897), 3 registres de recensement de la garde nationale mobile (1848-1870), 1 registre de déclaration d'ouverture d'écoles 
primaires et de salles d'asile (1886-1917), 122 planches cadastrales (18431846).

\section{Expositions}

Contribution à l'exposition " Chemins de mémoire, les Manceaux dans la Grande guerre " présentée à la médiathèque Louis Aragon du Mans.

Présentation d'une exposition dans l'enceinte des Archives du Mans sur une déclinaison de l'exposition citée ci-dessus, portant sur la mise en lumière d'une collection de factures et de courriers à en-tête.

\section{Publications}

Deux articles rédigés par Sophie Rouyer, directrice des Médiathèques et Archives du Mans, sont parus dans la revue La Vie Mancelle et Sarthoise : « La place de L'Éperon au xx $x^{\mathrm{e}}$ siècle "; " La place de l'Éperon, de la fortification défensive à la place publique » 